\title{
Thermal Effects in the Long-Term Monitoring of Bridges
}

\author{
Olivier L. Burdet \\ $\mathrm{PhD}$ \\ Ecole Polytechnique Fédérale \\ de Lausanne (EPFL), \\ Lausanne, Switzerland \\ olivier.burdet@epfl.ch
}

\author{
Olivier Burdet, born 1960, received \\ his civil engineering degree from the \\ Ecole Polytechnique Fédérale de \\ Lausanne, Switzerland and his $\mathrm{PhD}$ in \\ Structural Engineering from the \\ University of Texas at Austin. He is \\ currently lecturer and scientist at \\ EFPL. His interests are in the \\ monitoring of structures, e-learning, \\ the behavior of concrete structures \\ and conceptual design.
}

\begin{abstract}
Summary
Daily and seasonal temperature variations induce deflections of bridges. The amplitude of these moments is significant in comparison with the yearly increment caused by irreversible long-term deflections. To improve the quality of the interpretation of monitoring measurements, thermal movements thus need to be taken into account in the long-term monitoring of bridge deflections.

The paper presents the results of the long-term monitoring of deflections of several bridges in Switzerland over the past 20 years, with a particular attention given to thermal effects. The daily and seasonal movements of bridges under thermal effects are shown. The correlation between the measured temperatures and gradients and the position of bridge is shown, and methods to compensate for thermal effects are outlined. This allows to filter out the largest part of the thermal effects and to make more accurate assessments of the actual irreversible deformations.

The significant influence of temperature on the measurement results is illustrated on the basis of a large number of manual measurements by hydrostatic leveling and automatic measurements by electronic inclinometers. The simultaneous measurement of the ambient temperature and of the temperature of the concrete of the bridge itself gives useful information to take thermal effects into account.
\end{abstract}

Keywords: thermal effects, monitoring, bridge, deflection

\section{Introduction}

Monitoring of bridges has become an important component of the safety and maintenance setup of transportation lines. While the monitoring systems themselves can be compensated so that they are not sensitive to temperature changes, the monitored structure cannot. During the measurements, it is continuously exposed to thermal effects, mostly from the ambient temperature and direct sunlight, but also from wind or water-induced thermal fluxes. While these effects have long been known of specialists of the monitoring of structures, who, for example, often tend to perform their measurements during the night or at dawn to avoid the effects of direct sunlight, this type of approach may not always be sufficient to avoid all thermal effects. Additionally, some measurement methods, as for example surveying techniques or accurate GPS localization, need some time for the measurement to be performed [1]. This means that the measurement starts while the structure has a given shape, and ends when the structure has another shape, possible intermediate measurements being taken as the structure is in other, intermediate shapes. 
The present paper presents the theoretical bases of thermal deflections of bridges, then shows examples of measurements impacted by thermal effects and proposes some solutions to account for some of these effects.

\section{Thermal effects in the superstructure}

Thermal effects usually act differently on the top and bottom surface of bridge girders. Depending on the exposition to the sun, the height of the bridge and the nature of the terrain underneath (soil, water, etc.) Actual thermal profiles measured in bridges exhibit complex shapes as shown in Figure $0[2,3]$. For the calculation of deformations related to thermal effects, an equivalent linear temperature profile can be assumed [4]. This thermal profile induces a corresponding strain profile over the depth of the girder, resulting in a thermal curvature $\chi_{\text {therm }}$.

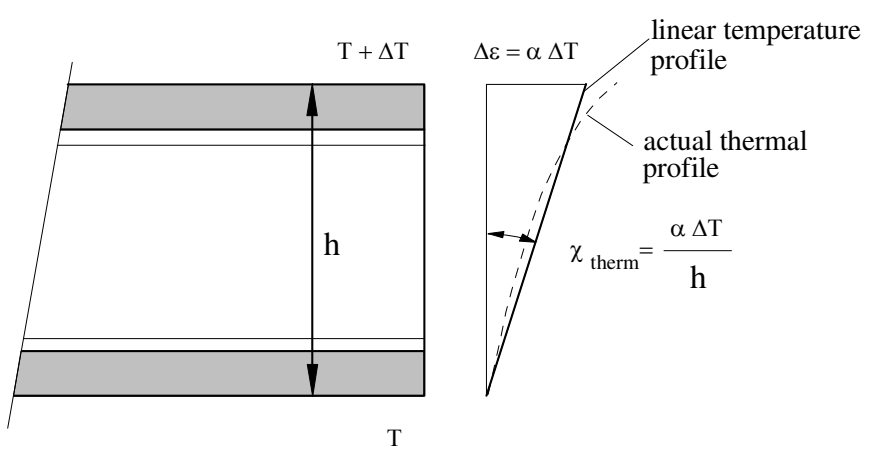

Fig. Error! Bookmark not defined.: Actual and linearized thermal profile over the height of a cross section and resulting curvature

For a simple beam with a constant cross section, a linear thermal profile constant over the length of the span produces a circular deflected shape, with a mid-span deflection of

$w_{\Delta T}=\alpha \cdot \frac{\Delta T}{h} \cdot \frac{\ell^{2}}{8}$

It can be observed that the relative deflection $w / \ell$ is proportional to the slenderness $\ell / \mathrm{h}$ of the beam and independent from the span:

$$
\frac{w_{\Delta T}}{\ell}=0.125 \cdot \alpha \cdot \Delta T \cdot \frac{\ell}{h}
$$

Table 1 shows the thermal deflections resulting from a linear gradient of $5^{\circ} \mathrm{C}$ between the top and bottom faces of the girder. This temperature difference is not the maximum possible value [3], but rather a value that can be expected on a typical sunny day. These thermal deflections are small compared to the deflections requirements for bridges in most countries, which limit the deflection to approximately $w / \ell \leq 1 / 1000$. But for monitoring purposes, these deflections are not so small: if the aim of the monitoring is to follow the evolution of the long-term deflections of a bridge, one can expect that these deflections will occur over several decades. Assuming a bridge in which the maximum deflection $w / \ell=1 / 1$ '000 will be reached after only 10 years and that the initial deflections is zero, then the yearly increase in deflection of the bridge is $\Delta \mathrm{w} / \ell=1 / 10$ ' 000 , which is comparable to the values of table 1 , which can occur in a single day.

This is why a good knowledge of thermal movements is essential to a quality monitoring, to avoid that thermal effects mask small-amplitude deflection increases. 
Table 1: Thermal deflections of a primatic simple beam subjected to a linear gradient of $5^{\circ} \mathrm{C}$

\begin{tabular}{|l|c|c|c|c|c|}
\hline Slenderness & 10 & 15 & 20 & 25 & 30 \\
\hline$w_{\Delta \mathrm{T}} / \ell$ & $1 / 16^{\prime} 000$ & $1 / 10^{\prime} 700$ & $1 / 8^{\prime} 000$ & $1 / 6^{\prime} 400$ & $1 / 5^{\prime} 300$ \\
\hline
\end{tabular}

Clearly, not all bridges are simple spans. When a system is statically indeterminate, the effect of thermal strains leads to a self-equilibrated state of stresses, in which deflections are smaller. Figure 1 shows the effect of an identical thermal gradient on a single beam and on a continuous beam with 2, 3, 4 and 5 spans (the length of the side spans being always 0.8 times the main span). The thermal deflections clearly concentrate on the side spans, with an amplitude approximately equal to only a quarter of that of the simple beam. Although considerably smaller, the thermal deflections in the intermediate spans are not completely negligible, when compared to the anticipated yearly deflections. Also of interest is the case of the double cantilever, which is, for example, the case of bridges built by the balanced cantilever method with a hinge at mid-span. In this case, the deflection is exactly the same than for a simple beam, but in the opposite direction.

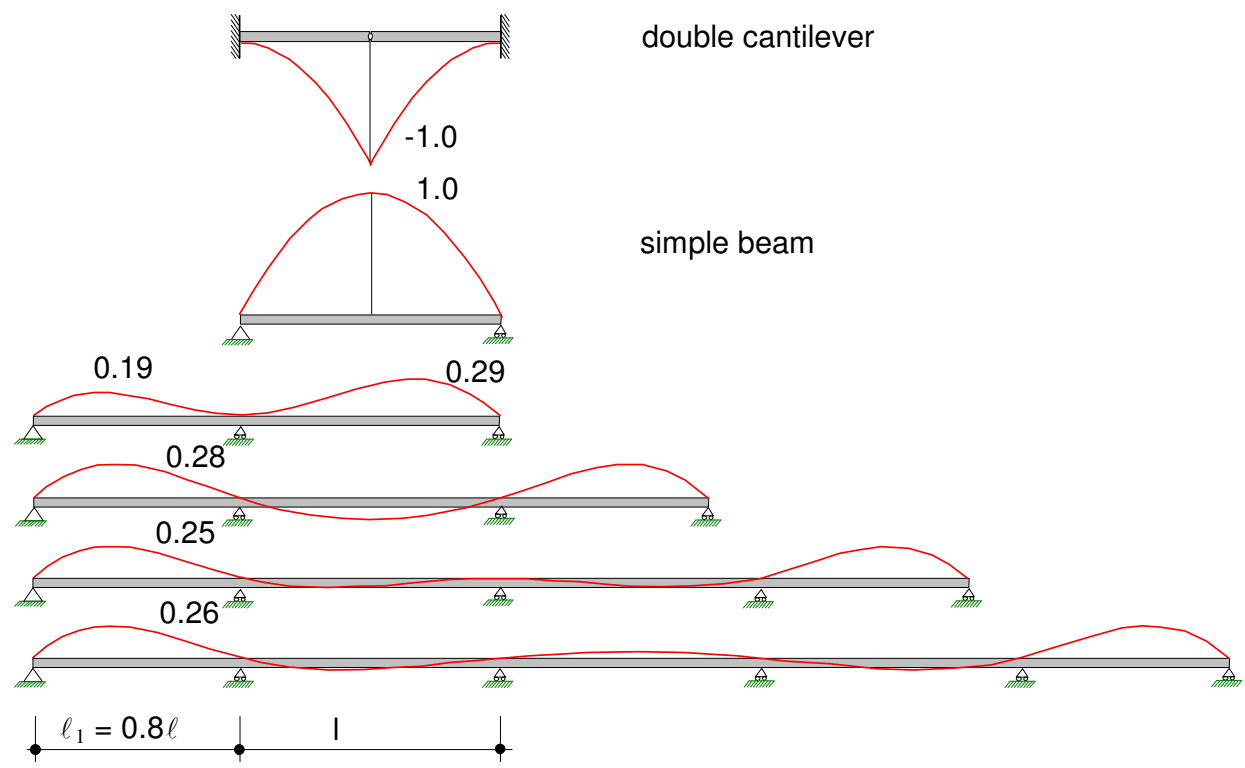

Fig. 1: Thermal deflections of a cantilever beam, a simple beam and of a continuous beam over 2, 3, 4 or 5 spans (beams with constant cross section)

It must also be noted that thermal effects to not only affect the superstructure of bridges, but also their infrastructure, namely bridge piers. For bridges with supports at the top of the piers, the effect of thermal expansion of the piers is almost negligible. For monolithic bridges, on the contrary, thermal effects causing a curvature of the pier (at sunrise or sunset) propagate to the girder, and can cause deflections of the same order of magnitude than thermal effects on the superstructure.

\section{Observed thermal deflections}

This section presents several examples of thermal movements of bridges, observed either during short-term measurements, such as loading tests, or during the long-term monitoring of structures.

\subsection{The Lutrive bridges}

The Lutrive bridges are twin bridges built in the early 70's by the balanced cantilever method, with cast-in-place elements. The particularity of these bridges is that they have a hinge at mid-span, which makes the system almost statically determinate. As a consequence, the bridges are quite 
sensitive to thermal effects. Figure 2(a) shows the evolution of the mid-span deflection over time. The initial part of the measurements were performed by a surveying team with intervals of approximately 5 years. Starting in 1988, measurements were taken three times per year using an hydrostatic levelling system permanently installed in the bridge [5,6,7]. It can immediately be seen that the more frequent measurements did not make following the bridge's deflections easier: with more measurements came more thermal effects, and an increased scatter of the measurements. As Fig. 2(b) shows, the daily amplitude of the deflections is quite large (approximately $20 \mathrm{~mm}$ ). It was only by pursuing the measurements over an extended period of time that a proper vision was gained on the long-term tendency for the deflections. This led, at the end of the 1990s, to a second additional prestressing in the South Bridge. The measurements clearly showed that an additional strengthening of the North Bridge was not necessary.

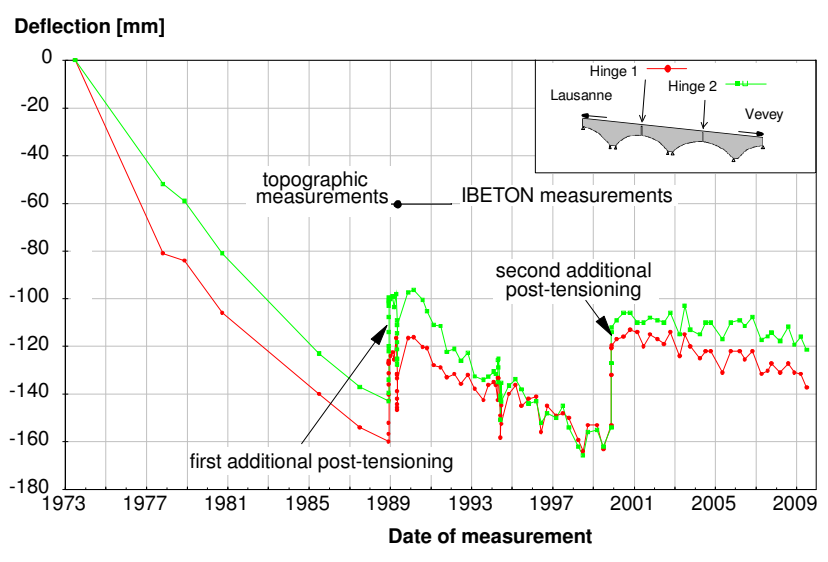

(a) Long-term defections since the end of the construction

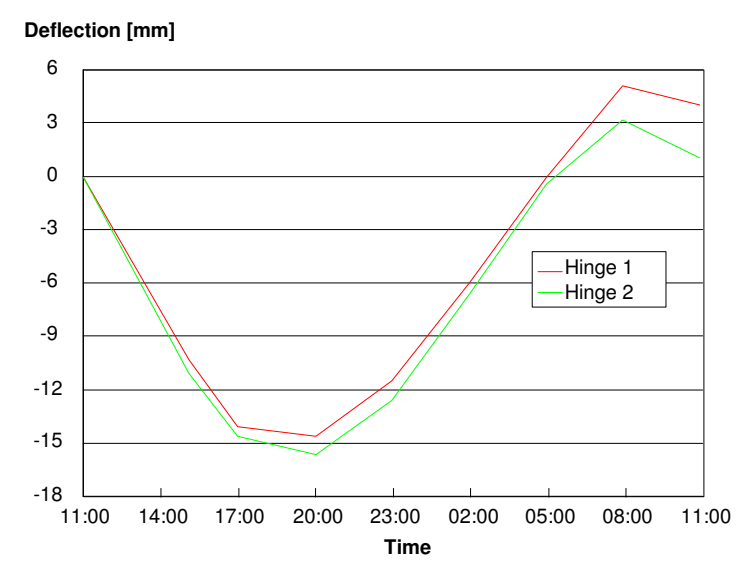

(b) Daily movements

Fig 2: Deflections of the Lutrive South Bridge

\subsection{The Mentue bridges}

The Mentue bridges are also twin bridges, but they were built more recently, with a totally monolithic system, except at the abutments. The measurements performed on that bridge during a period of 24 hours show that the structure also undergoes thermal movements, although with an amplitude approximately two times smaller than the Lutrive Bridges, for comparable spans. This is in spite of the fact that the Mentue bridges are monolithic whereas the Lutrive Bridges have a hinge at mid-span.

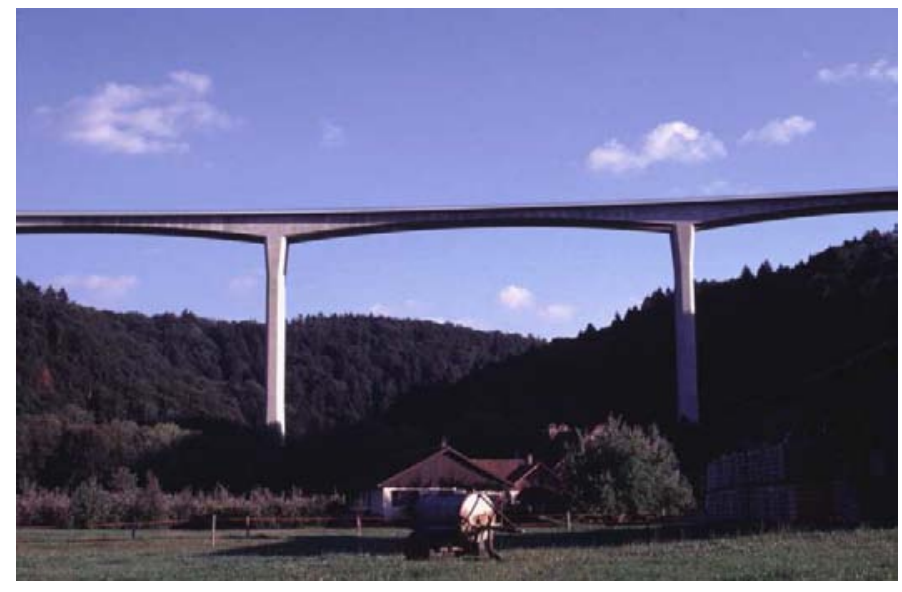

(a) General view

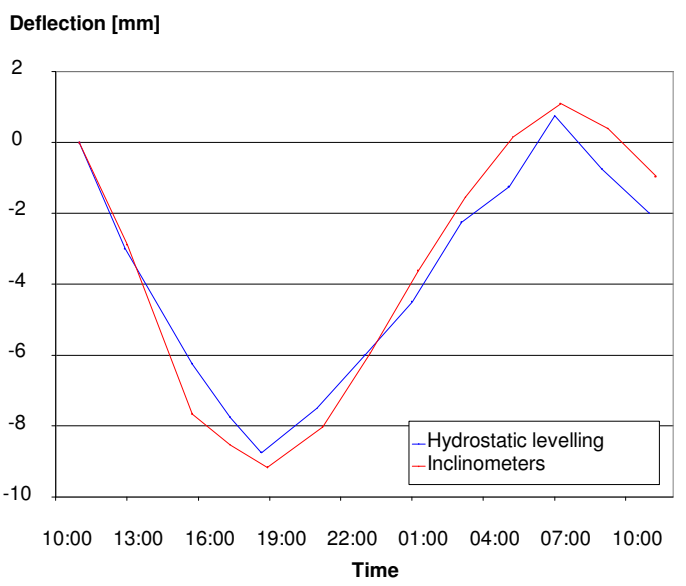

(b) Deflections measured over 24 hours

Fig. 3: Mentue Bridges 


\subsection{The Riddes bridges}

The Riddes Bridges are twin highway bridges built in the late 1980's. Because of their peculiar shape, they were also equipped with a hinge at their mid-span. This has for consequence that the bridges behave almost as consoles, with a great sensitivity to thermal effects. These two bridges were monitored for a total of 4 years to follow their mid-span deflections that, according to some measurements by surveyors, was thought to be increasing at a rapid pace. Soon after the installation of the monitoring system, it was recognized that the particular geometry and statical system of the Riddes bridges make them extraordinarily sensitive to thermal effects $[8,9]$. This is in addition to being located in one of the sunniest parts of Switzerand.

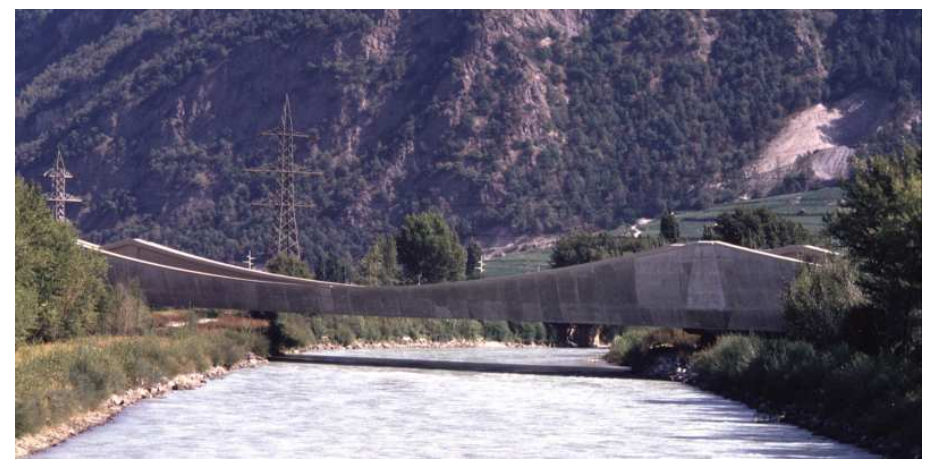

Fig. 4: General view of the Riddes Bridges over the Rhone River

Figure 5 shows an example of the recorded temperature variations of the Riddes Bridge over a period of 10 days and 2 months respectively. During that time period, no significant work was performed on the bridge and measurements influenced by the traffic are filtered out, so all observed movements are solely caused by thermal effects. The figure also shows the measured ambient temperature underneath the bridge for the same period of time. Amongst the numerous available temperature measurements, this was found to be the most reliable and the most easily connectable to the bridge's movements. It can be easily recognized that the daily amplitude of the temperature variations directly translates into daily movements. From the measurements over two months, it can be observed that periods when the daily temperature amplitude was smaller correspond to periods where the bridge daily amplitudes are smaller, and the bridge is in a higher position. On the contrary, days when the temperature variation was larger correspond to lower positions of the bridge and larger daily movements. Over the period considered, the maximum daily amplitude of the deflections is approximately 40 to $45 \mathrm{~mm}$, which is 1/ 3'300. The long-term deflection that needed to be identified was in the order of 2 to $3 \mathrm{~mm} /$ year.
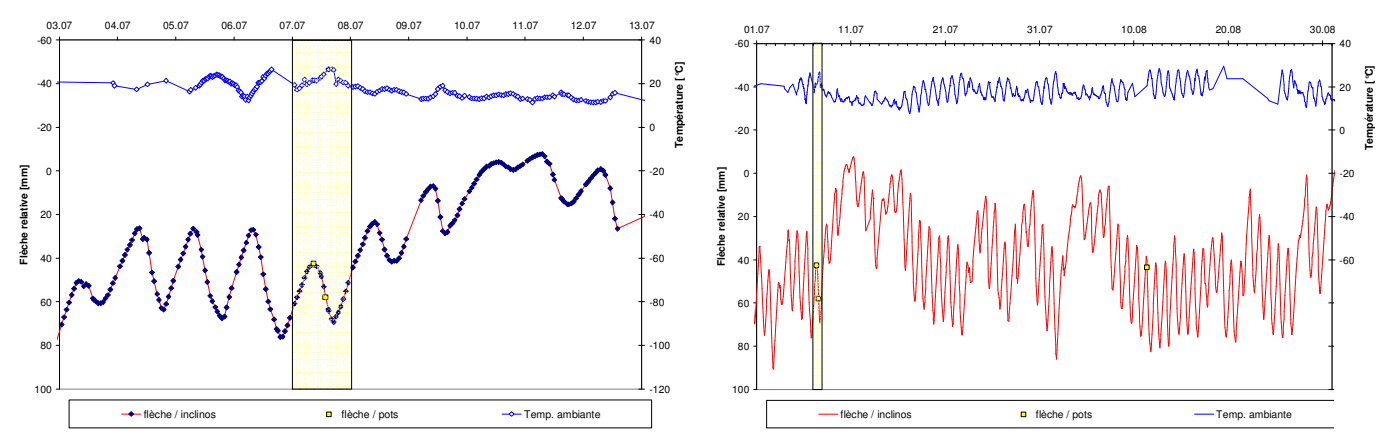

Fig. 5: Mid-span deflection of the Riddes bridge over a period of 10 days and 2 months 


\section{Cancellation of thermal deflections}

As was just shown, the effect of thermal deflections can be large, and its importance must be assessed and recognized in monitoring project. In some cases, as in the case of the Lutrive bridges, it is sufficient to take it into account and to wait until a sufficient number of measurements are available to be able to discern the long-term tendency of the deflections.

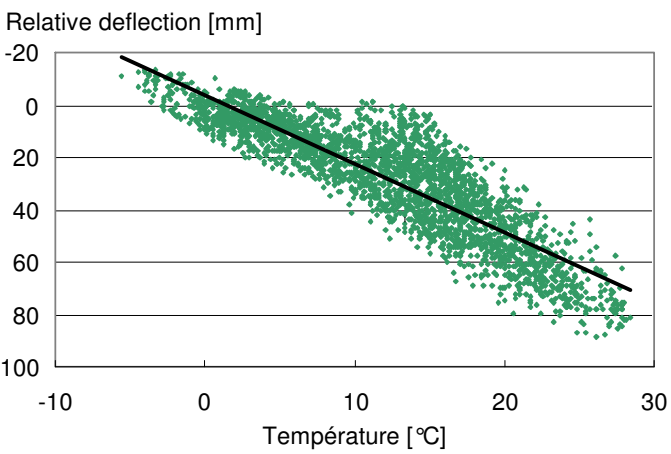

(a) 2000

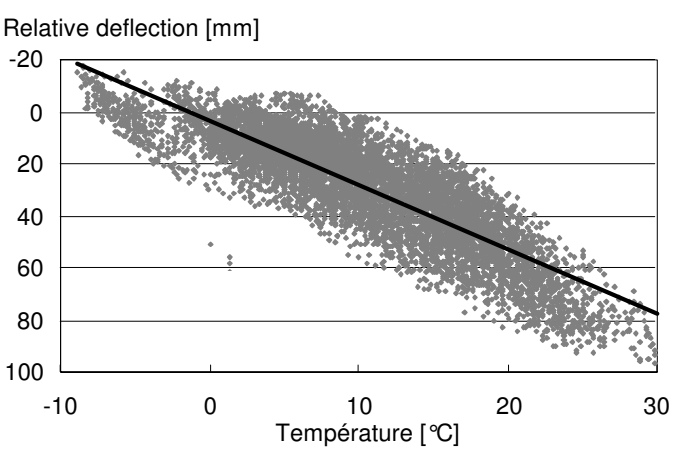

(c) 2002

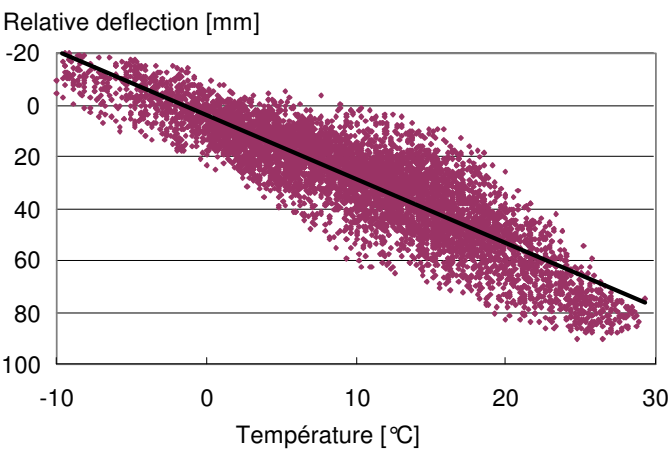

(b) 2001

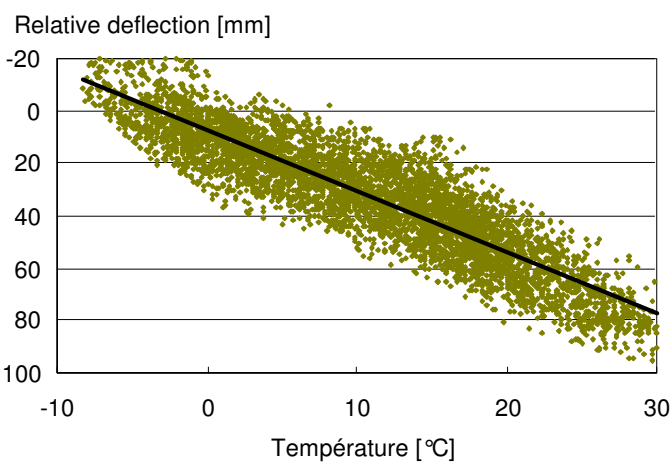

(d) 2003

Relative deflection [mm]

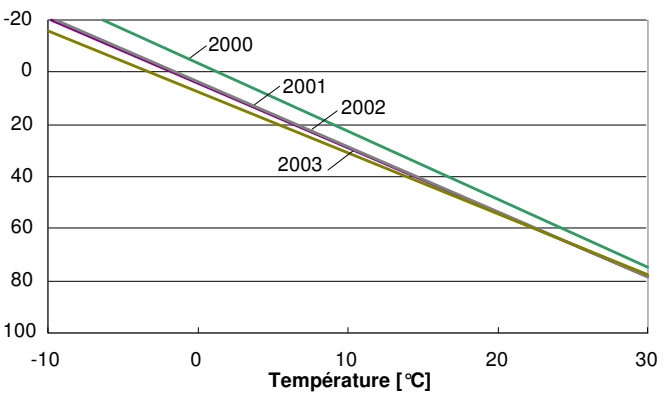

(e) Comparison of the regression lines

Fig. 6: Relative deflection as a function of the temperature for the Riddes South Bridge over four consecutive years and corresponding regression lines

In other cases, as in the case of the Riddes bridges, it was necessary to be able to rapidly assess the long-term tendency of the deflections. In this case, to be able to reach consistent conclusions, it was necessary to cancel out the effect of thermal deflections to be able to make a proper assessment. This was performed by representing the yearly measurement results in a temperature versus deflections graph, shown in Fig. 6. For each measurement year, representing approximately the same number of measurements and the same range of temperatures, a regression line was calculated, representing the average relationship between the measured ambient temperature and the corresponding bridge deflections. Subsequently, these regression lines were compared to one 
another. As can be seen, the regression lines change very little form one year to the following, with almost the same slope and only small downward increments. This allowed concluding that the bridges' deflection increases only by 2.5 to $3 \mathrm{~mm}$ per year.

A direct compensation of each measurement as a function of the measured deflection and temperature values was not performed. In view of the complexity of the observed behavior, it is not certain that this would be possible, although some partial compensations showed promising results. In this case, a yearly averaged value was sufficient.

\section{Conclusions}

Thermal deflections of bridges are unavoidable during the monitoring. Careful planning and some continuous observations of the behavior over a period of at least one day can allow identifying the thermal sensitivity of the observed bridge. The initial measurement results need to be considered with care, until a good understanding of the various influences has been gained.

Temperature measurements should always be taken in parallel to the measurement of deflections. In the case of manual measurements, care should be taken to perform them as much as possible each under the same circumstances (same period of the year, same time of the day), to minimize as much as possible thermal effects. In addition, note should be taken of the ambient temperature and weather condition, to help understanding the measurements when they are compiled.

\section{References}

[1] BURDET O., MUTTONI A., Evaluation des systèmes existants pour le suivi à long terme des déformations des ponts, Office fédéral des routes, $\mathrm{N}^{\circ}$ 607, 57 p., Bern, Switzerland, French, 2006.

[2] PRIESTLEY M. J. N., Design of concrete bridges for temperature gradients, ACI Journal, vol 75 n5, pp. 209-217, New Zealand, 1978.

[3] LUCAS J.-M., VIRLOGEUX M., LOUIS C., Temperature in the Box Girder of the Normandy Bridge, Structural Engineering International, IABSE, 2005/3, pp. 156-165, Zürich, Switzerland, 2005.

[4] SIA, SIA 261 - Actions sur les structures porteuses, 114 p., Zürich, Switzerland, 2003.

[5] FAVRE R., CHARIF H., MARKEY I. F., Observation à long terme de la déformation des ponts, Rapport OFR, 86/88, Switzerland, 1990.

[6] FAVRE R., MARKEY I. F., Long-term monitoring of bridge deformation, Bridge Evaluation, Repair and Rehabilitation. A. S. Nowak (ed.) NATO ASI series E. Kluwer academic Publishers, Dordrecht, 187, pp. 85-100, Baltimore, USA, 1990.

[7] BURDET O., BADOUX M., Long-term deflection monitoring of prestressed concrete bridges retrofitted by external post-tensioning - examples from Switzerland, IABSE Rio 1999, Rio de Janeiro, Brazil, English, 1999.

[8] BURDET O., ZANELLA J.-L., Automatic Monitoring of Bridges using Electronic Inclinometers, IABSE, , Lucerne Congress Structural Engineering for Meeting Urban Transportation Challenges, pp. 398-399, Zürich, Switzerland, 2000.

[9] BURDET O., ZANELLA J.-L., Automatic Monitoring of the Riddes Bridges using Electronic Inclinometers, IABMAS, First International Conference on Bridge Maintenance, Safety and Management, Barcelona, Spain, 2002. 